\title{
SIMPLIFYING THE STRUCTURE OF SECOND ORDER PARTIAL DIFFERENTIAL EQUATIONS ${ }^{(1)}$
}

\author{
BY \\ AVNER FRIEDMAN
}

1. The problem. Consider the differential equation

$$
\Delta_{2} A+b^{i} A_{i}+c A=0
$$

where the summation convention is being used and

$$
\Delta_{2} A=\frac{1}{g^{1 / 2}} \frac{\partial}{\partial x^{i}}\left(g^{1 / 2} g^{i j} \frac{\partial A}{\partial x^{i}}\right)=g^{i j} A_{, i j}=g^{i j}\left(A_{. i j}-\Gamma_{i j}^{k} A_{k}\right)
$$

is the Laplace-Beltrami operator. Here a comma indicates covariant differentiation, a dot indicates (ordinary) differentiation, $A_{i}=A_{\cdot i}, g=\operatorname{det}\left(g_{i j}\right)$ and is always assumed to be positive, and $\Gamma_{i j}^{k}$ are the Christoffel symbols of the second kind with respect to $\left(g_{i j}\right)$. All the functions in this paper are assumed to be sufficiently smooth. If (1) has a positive solution $A_{0}$ in a domain $D$, then the structure of (1) can be simplified. Indeed, setting $A=A_{0} \bar{A}$, we reduce (1) to

$$
\Delta_{2} \bar{A}+b^{i} \bar{A}_{i}=0
$$

where the $b^{i}$ depend on $b^{i}, A_{0}$.

In this paper we consider the equation

$$
\Delta_{2} A+c A=0
$$

and wish to obtain an equivalent equation

$$
\bar{\Delta}_{2} \bar{A}+c_{0} \bar{A}=0
$$

where $c_{0}$ is a constant, preferably zero. Here, $\bar{\Delta}_{2}$ is the Laplace-Beltrami operator with respect to a different metric. We shall prove that it is possible to reduce (4) to (5), and that we can take $c_{0}=0$ if $c \leqq 0$. We also get similar results for parabolic equations.

Ishii [6] has considered conformal mappings which transfer solutions of $\Delta_{2} A=0$ into solutions of $\bar{\Delta}_{2} \bar{A}=0$. Ingraham [4] considered the question of eliminating the $b^{i}$ from equation (1) when $c \equiv 0$, on account of replacing the

This paper has been submitted to and accepted for publication by the Proceedings of the American Mathematical Society. It has been transferred to these Transactions, with the consent of the author, for technical reasons. Received by the editors July 20, 1960 and, in revised form, September 22, 1960.

(1) Prepared under Contract Nonr 710(16)(NR 044 004) between the Office of Naval Research and the University of Minnesota. 
covariant derivatives in $\Delta_{2}$ by covariant derivatives with respect to some nonRiemannian affine connections. In [5] he considered the parabolic case.

2. The transformation. We try to perform conformal mapping

$$
\bar{g}_{i j}=e^{2 \sigma} g_{i j}
$$

and set

$$
\bar{A}=e^{h(\sigma)} A
$$

where $\sigma$ and $h(\sigma)$ are to be determined. Using the formulas [3, p. 89]

$$
\begin{aligned}
& \bar{g}^{i j}=e^{-2 \sigma} g^{i j}, \\
& \bar{\Gamma}_{i j}^{k}=\Gamma_{i j}^{k}+\delta_{i}^{k} \sigma_{j}+\delta_{j}^{k} \sigma_{i}-g_{i j} g^{k m} \sigma_{m}
\end{aligned}
$$

we find, after some elementary calculation,

$$
\begin{aligned}
\bar{\Delta}_{2} \bar{A}+c_{0} \bar{A}=e^{-2 \sigma} e^{h(\sigma)}\left\{\left(\Delta_{2} A+c A\right)+\left(2 h^{\prime}(\sigma)+n-2\right) g^{j k} \sigma_{j} A_{k}\right. \\
\left.+\left[h^{\prime \prime}(\sigma) \Delta_{1} \sigma+h^{\prime}(\sigma) \Delta_{2} \sigma+h^{\prime}(\sigma)\left(h^{\prime}(\sigma)+n-2\right) \Delta_{1} \sigma-c+e^{2 \sigma} c_{0}\right] A\right\}
\end{aligned}
$$

where $\Delta_{1} \sigma=g^{i j} \sigma_{i} \sigma_{j}$. Hence, (4) and (5) are equivalent in any subdomain of $D$ if and only if the coefficients of $A$ and $A_{k}$ vanish. The vanishing of the coefficients of the $A_{k}$ is equivalent to

$$
h(\sigma)=\frac{2-n}{2} \sigma .
$$

Assuming $n \geqq 3$ we find that the coefficient of $A$ vanishes if and only if

$$
\Delta_{2} \sigma+\frac{n-2}{2} \Delta_{1} \sigma+\frac{2 c}{n-2}-\frac{2 c_{0}}{n-2} e^{2 \sigma}=0 .
$$

Setting

$$
u=\exp \left\{\frac{n-2}{2} \sigma\right\}
$$

(10) becomes

$$
\Delta_{2} u+c u=c_{0} u^{(n+2) /(n-2)}, \quad u>0 \text {. }
$$

We have thus proved:

TheOREM 1. Let $n \geqq 3$. Equation (4) is reducible in a domain $D$ to equation (5) by means of the conformal transformation (6), (7) if and only if equation (12) has a positive solution in $D$.

Due to the invariance of the operators of (4), (5) with respect to local change of the $x$-coordinates, Theorem 1 holds also when $D$ is a domain on a manifold. 
Quite incidentally, if $c$ and $c_{0}$ are the scalar curvatures corresponding to the metric tensors $g_{i j}$ and $\bar{g}_{i j}$ related by (6), then (10) is known to hold (see [3, p. 90]). Recently Yamabe [9] proved that on a compact manifold, for any positive definite metric there exists a conformal transformation which yields a new metric with constant scalar curvature. His proof does not make use of the specific nature of the function $c$, that is, he proves that for any smooth function $c$ on a compact Riemannian manifold with a positive definite metric there exists a constant $c_{0}$ and a positive smooth function $u$, defined on the whole manifold, such that (12) is satisfied. Hence we have:

Theorem 2. Given a compact Riemannian manifold $R_{n}, n \geqq 3$, with a positive definite metric, equation (4) can globally be reduced to equation (5) by means of (6), (7), (9), where $c_{0}$ is a constant depending on $c$.

If $c_{0} \leqq 0$ then the transformation is uniquely determined, up to a constant multiple $\neq 0$. Indeed, if $u_{1}, c_{1}$ is another solution, then $w=u_{1} / u$ satisfies $\bar{\Delta}_{2} w+c_{0} w=c_{1} w^{(n+2) /(n-2)}$. Setting $w\left(x^{0}\right)=\max w(x)$, we then have $\bar{\Delta}_{2} w \leqq 0$, $c_{0} w \leqq 0$ at $x^{0}$; hence $c_{1} \leqq 0$. It follows that $\bar{\Delta}_{2} w+c_{0} w \leqq 0$. The minimum principle now yields $w \equiv$ const.

Corollary. Let $D$ be an $n$-dimensional bounded domain, $n \geqq 3$, and let $\left(g_{i j}\right)$ be a positive matrix. Then (4) is reducible to (5), in the whole domain $D$, by means of (6), (7), (9), where the constant $c_{0}$ depends on $c$.

Under some conditions on $c(x)$ we can even take $c_{0}=0$. Thus we have:

Theorem 3. Let $D$ be an $n$-dimensional domain, $n \geqq 3$, either bounded or unbounded but with finite boundary, and let $\left(g_{i j}\right)$ be a positive matrix. If $c(x) \leqq 0$ then (4) is reducible, in the whole domain $D$, to (5) with $c_{0}=0$, by means of $a$ transformation (6), (7), (9).

Proof. We only have to establish the existence, in $D$, of a positive solution of the equation

$$
\Delta_{2} u+c u=0 .
$$

If $D$ is bounded, we solve (13) for any positive boundary values and thus obtain a positive solution, using the maximum principle. If $D$ is unbounded then the existence of a positive solution follows by recent results of Meyers and Serrin [7].

We note that if the diameter of $D$ is sufficiently small it is not necessary to make any assumption on $c$. We also remark that for a given bounded domain $D$ we can get $c_{0}=0$ if $c(x) \leqq \epsilon$ where $\epsilon$ is sufficiently small, depending on $D$. However, if $\epsilon$ is not small there are in general no positive solutions of (13) in $D$.

For simplicity, we produce a counter-example for $n=3, \Delta_{2}$ being the Laplace operator $\Delta$, and $c(x) \equiv k^{2}>0$. Let $x^{0}$ be a point in $D$ whose distance from 
the boundary of $D$ is $H$. We claim that there are no positive solutions of $\Delta u+k^{2} u=0$ in $D$ if $k>\pi / H$.

Indeed, we apply the Pizetti formula $[1$, p. 259]

$$
\frac{1}{4 \pi R^{2}} \int_{S_{R}} u(x) d S=\sum_{h=0}^{m} \frac{R^{2 h} \Delta^{h} u\left(x^{0}\right)}{(2 h+1) !}+\frac{1}{4 \pi(2 m+1) !} \int_{K_{R}} \frac{(R-r)^{2 m+1} \Delta^{m+1} u(x)}{R r} d V
$$

where $K_{R}$ is a ball of radius $R$, center $x^{0}$ and surface $S_{R}$, and $r=\left|x-x^{0}\right|$. Taking $m \rightarrow \infty$ we obtain

$$
\frac{1}{4 \pi R^{2}} \int_{S_{R}} u(x) d S=\sin (k R) u\left(x^{0}\right) / k R .
$$

Now, if $u$ is positive in $D$ then it follows that $\sin k R>0$; hence $k H \leqq \pi$ which is a contradiction.

THE CASE $n=2$. From (8) we conclude that a reduction of (4) to (5) is possible if $h(\sigma)=0$ and if

$$
c=e^{2 \sigma} c_{0} .
$$

Hence, the reduction by (6), (7) is possible if and only if $\operatorname{sgn} c(x)=$ const. and $c_{0}$ can be taken to be either any positive or any negative number, depending on sgn $c(x)$. Note that $\left(g_{i j}\right)$ can be taken to be any nonsingular matrix.

3. Parabolic equations. Consider the parabolic equation

$$
a \frac{\partial A}{\partial t}=\Delta_{2} A+c A
$$

where $x$ varies in an $n$-dimensional domain $D(n \geqq 3)$ and $0 \leqq t<\infty$. The coefficients are functions of $(x, t)$ and $\Delta_{2}$ is elliptic. Performing the transformation (6), (7), (9) with $\sigma$ depending also on $t$, we find that (15) is reduced to

$$
\bar{a} \frac{\partial \bar{A}}{\partial t}=\bar{\Delta}_{2} \bar{A}+c_{0} \bar{A}
$$

if and only if there exists a positive solution of

$$
a \frac{\partial u}{\partial t}=\Delta_{2} u+c u-c_{0} u^{(n+2) /(n-2)}
$$

and then $\sigma$ is defined by (11). Also,

$$
\bar{a}=e^{-2 \sigma} a .
$$

From now on we assume that if $D$ is unbounded then the functions $a, 1 / a$, $c, g_{i j}, g^{i j}, \partial a / \partial x^{i}, \partial^{2} a / \partial x^{i} \partial x^{i}, \partial a / \partial t, \partial g^{i j} / \partial x^{h}, \partial^{2} g^{i j} / \partial x^{h} \partial x^{k}, \partial g^{i j} / \partial t$ are Hölder continuous and bounded for $x$ in $D$ and $t$ in finite intervals. We then claim 
that for any $D$, there exists a positive solution of (17) with $c_{0}=0$. It is enough to establish it when $D$ is the whole $n$-dimensional space $E_{n}$.

Let $K(x, t ; \xi, \tau)$ be the fundamental solution of (17) constructed by Dressel [2]. Then for any positive function $\phi(\xi)$ which tends to a positive limit $B$ as $|\xi| \rightarrow \infty$ we have a solution of (17) with $c_{0}=0$ for $x \in E_{n}, 0<t<\infty$, in the form

$$
u(x, t)=\int_{E_{n}} K(x, t ; \xi, 0) \phi(\xi) d \xi
$$

This solution is positive on $t=0$, and it tends to $B$ as $|x| \rightarrow \infty$, uniformly in $t$ in finite intervals $0 \leqq t \leqq T, T>0$. Hence by appropriately applying the maximum principle [8] we conclude that $u(x, t)>0$. We have thus proved:

Theorem 4. Let $D$ be any domain in $E_{n}, n \geqq 3$ and let the coefficients of the parabolic equation (15) satisfy the boundedness assumptions mentioned above. Then equation (15) is reducible to equation (16) with $c_{0}=0$, by means of (6), (7), (9).

Note that no assumption is being made on the signature of $c$.

If $n=2$, we first make a transformation of the form $\hat{A}=e^{\alpha t} A$ and obtain a new coefficient $c$ which is positive. We then can reduce (15) to (16) since equation (14) can then be solved.

\section{REFERENCES}

1. R. Courant and D. Hilbert, Methoden der mathematischen Physik, vol. 2, Berlin, Julius Springer, 1937.

2. F. G. Dressel, The fundamental solution of the parabolic equation, Duke Math. J. vol. 7 (1940) pp. 186-203.

3. L. P. Eisenhart, Riemannian geometry, Princeton, Princeton University Press, 1949.

4. R. L. Ingraham, The geometry of the linear partial differential equation of the second order, Amer. J. Math. vol. 75 (1953) pp. 691-698.

5. - The geometry of the heat equation, Compositio Math. vol. 12 (1954) pp. 147-156.

6. Y. Ishii, On conharmonic transformation, Tensor vol. 7 (1957) pp. 73-80.

7. N. Meyers and J. B. Serrin, The exterior Dirichlet problem for second order linear elliptic equations, J. Math. Mech. vol. 9 (1960) pp. 513-538.

8. L. Nirenberg, $A$ strong maximum principle for parabolic equations, Comm. Pure Appl. Math. vol. 6 (1953) pp. 167-177.

9. H. Yamabe, On a deformation of Riemannian structures on compact manifolds, Osaka Math. J. vol. 12 (1960) pp. 21-37.

University of Minnesota, Institute of Technology, Minneapolis, Minnesota 